\title{
Un « chaos linguistique » : les textes en français d'Amelia Rosselli (1930-1996)
}

\section{Emilio Sciarrino}

\section{OpenEdition}

\section{Journals}

Édition électronique

URL : http://journals.openedition.org/coma/311

DOI : 10.4000/coma.311

ISSN : 2275-1742

Éditeur

Institut des textes \& manuscrits modernes (ITEM)

\section{Référence électronique}

Emilio Sciarrino, «Un « chaos linguistique » : les textes en français d'Amelia Rosselli (1930-1996)», Continents manuscrits [En ligne], 2 | 2014, mis en ligne le 22 avril 2014, consulté le 19 avril 2019. URL http://journals.openedition.org/coma/311 ; DOI : 10.4000/coma.311

Ce document a été généré automatiquement le 19 avril 2019

\section{(i)}

Continents manuscrits - Génétique des textes littéraires - Afrique, Caraîbe, dispora est mis à disposition selon les termes de la licence Creative Commons Attribution - Pas d'Utilisation

Commerciale - Pas de Modification 4.0 International. 


\title{
Un « chaos linguistique » : les textes en français d'Amelia Rosselli (1930-1996)
}

\author{
Emilio Sciarrino
}

«fleurs are not flowers » Amelia Rosselli, Diario in Tre Lingue ${ }^{1}$

1 L'œuvre poétique d'Amelia Rosselli est marquée par un rapport fondamental à la langue et à la culture française : un lien aussi fort, peut-être, que celui qui l'unit à l'anglais. De ses débuts en 1952 jusqu'à ses derniers poèmes, parus à titre posthume, son écriture reflète en permanence cette situation "entre les langues ${ }^{2}$ ». Sa langue n'est pas simplement l'italien mais un "ydioma tripharium ${ }^{3}$ » inextricablement lié à son parcours biographique.

2 Amelia Rosselli est née à Paris en 1930. Son père, Carlo Rosselli, était un militant antifasciste en exil, fondateur d'un célèbre mouvement de résistance socialiste, Giustizia e Libertà (Justice et Liberté). Sa mère, Marion Cave, était anglaise. Après l'assassinat de Carlo et de son frère Nello (Sabatino) Rosselli par la Cagoule sur l'ordre de Mussolini (1937), la famille Rosselli fuit à Londres, puis aux États-Unis, avant de revenir à Rome, où Amelia Rosselli s'installe définitivement.

3 À partir de 1952, Amelia Rosselli écrit en trois langues une série de poèmes : d'abord en anglais My Clothes to the Wind (Mes vêtements au vent), 1952, puis en italien Cantilena, poesie per Rocco Scotellaro (Cantilène, poésies pour Rocco Scotellaro), 1953, ainsi qu'en français Adolescence: Sanatorio 1954 (Adolescence: Sanatorium 1954), 1954. Enfin, dans Le Chinois à Rome, 1955, le français est la langue principale, mais les deux autres langues affleurent de manière décisive. De même, Diario in Tre Lingue (Journal en Trois Langues), 1955-1956, est écrit en trois langues à la fois 4 .

4 À la fin de cette expérience trilingue (onze ans d'écriture), Amelia Rosselli semble choisir l'italien comme langue d'écriture principale : la publication de Variazioni belliche Variations de guerre ${ }^{5}$ en 1964 marque le début d'une œuvre poétique surtout italophone, et qui justifie de fait son rattachement au canon littéraire italien. Mais ce choix ne saurait 
masquer l'utilisation constante des deux autres langues, qui se manifeste dès le premier poème de son recueil Variazioni belliche par l'insertion de mots français, anglais, ou bien par des néologismes d'origine étrangère.

5 À la présence d'autres langues au sein de l'écriture en italien s'ajoute une constante influence culturelle, qui peut s'exprimer par le choix de formes poétiques peu courantes dans la tradition italienne ( «long poem », poème en prose) ou bien par des références à des auteurs étrangers. Certes, les trois principaux recueils de l'auteure sont publiés en italien Variazioni belliche, 1964; Serie ospedaliera (Série hospitalière), 1969; Documento ( Document), 1976, mais le recueil trilingue Primi scritti (Premiers écrits) et le recueil de poèmes anglais Sleep $\left(\right.$ Sommeil $^{6}$ ) rappellent ce trilinguisme originel - ou triglossie, comme nous verrons - qui a toujours constitué le noyau poétique de l'écriture d'Amelia Rosselli.

6 L'audace d'ignorer ou de transgresser délibérément les frontières entre les langues et les littératures appartenant à des nations différentes ne pouvait passer inaperçue, ou encore moins être reconnue de manière univoque, sans susciter les discussions virulentes qui caractérisèrent la réception d'une œuvre controversée. Amelia Rosselli fut ainsi considérée comme un véritable "cas littéraire " à part. Il faut attendre nos jours pour qu'elle connaisse en Italie une canonisation confirmée par la publication de nombreuses études de son œuvre ${ }^{7}$. Pourtant, alors qu'Amelia Rosselli est sans cesse citée comme un modèle par les poètes de la génération actuelle, sa légitimité dans le corpus très restreint des classici del Novecento (classiques $\mathrm{du} \mathrm{xx}^{\mathrm{e}}$ siècle) est encore incertaine et parfois remise en cause. Non seulement les femmes qui s'imposent dans le champ poétique sont particulièrement rares en Italie ${ }^{8}$, mais la langue poétique d'Amelia Rosselli se heurte à toute conception linguistique normative et monolingue. Assourdie par son propre monolinguisme, la réception critique négligea le plus souvent la dimension plurilingue de la poésie d'Amelia Rosselli pour s'orienter vers d'autres types d'interprétations.

7 C'est dans ce contexte d'un inévitable hiatus entre multilinguisme personnel et «monolinguisme de l'autre " " qu'Amelia Rosselli emploie le français, d'abord comme langue d'écriture en soi, puis comme langue dans laquelle puiser des structures formelles et culturelles afin de les transposer en italien ou en anglais. Si dans la coprésence de plusieurs langues le français n'a pas une identité figée, il faut néanmoins lui reconnaître ses caractéristiques spécifiques. Pour Amelia Rosselli, le français est une langue d'élection pour des raisons biographiques et culturelles, et il occupe une place particulière dans ce système trilingue.

\section{Situation de la langue française dans l'œuvre d'Amelia Rosselli}

8 Le rapport d'Amelia Rosselli au français peut d'abord se définir par comparaison et par exclusion. Ce n'est pas un choix autonome, qui partirait d'une autre langue étrangère pour arriver au français en tant que langue d'élection (nous pensons à Cioran ou encore à Kundera). Au contraire, Amelia Rosselli a la langue française comme origine, en même temps que l'italien et l'anglais. Cette triple origine s'enracine profondément, toutefois, dans des conditions historiques et politiques bien déterminées. Amelia Rosselli refusait l'adjectif « cosmopolite» et se considérait comme faisant partie de la grande catégorie des « enfants de la Seconde Guerre mondiale ${ }^{10}$ ». Ce terme désigne la fracture traumatique qui a transformé la société intellectuelle européenne du "monde d'hier », avant la 
guerre, en une nouvelle communauté décentrée, marquée par les émigrations forcées, issue de la ruine des empires et de la chute des sociétés totalitaires.

Comme nous l'avons rappelé, Amelia Rosselli est née à Paris en 1930 où son père était réfugié, comme de nombreux exilés politiques italiens. Cette naissance dans l'exil a comme conséquence un apprentissage trilingue. Silvia Rosselli, cousine d'Amelia, décrit la situation familiale en ces termes : «À Paris nous avons toujours parlé un mélange de langues : italien avec papa et maman, anglais avec les nurses anglaises, français à l'école ${ }^{11}$ . » Elle précise aussi à propos d'Amelia : " On peut dire que d'une certaine manière elle n'a jamais eu une "langue maternelle ${ }^{12}$.» La jeune Amelia se trouve dans une situation de triglossie, chaque langue ayant un domaine spécifique d'utilisation relevant de la sphère sociale ou affective. Cependant, le terme de «mélange» suggère déjà un décloisonnement, une forme de nette continuité, voire d'interférence potentielle entre ces langues. De plus, selon Silvia Rosselli, cette multiplicité équivaut à l'absence d'une « langue maternelle » unique. Cette absence d'une langue d'origine est exacerbée par des souvenirs traumatiques, puisque le père et l'oncle d'Amelia Rosselli furent assassinés avec une extrême violence en 1937. Ce meurtre est un thème récurrent dans ses poèmes, bien que de manière cryptée ou implicite: Amelia Rosselli l'évoque pour la première fois en français ${ }^{13}$.

10 La langue et la littérature française représentent aussi un vaste héritage culturel auquel Amelia Rosselli se réfère fréquemment. Paris et la littérature française bénéficient à cette époque d'un prestige remarquable, en particulier en Italie $^{14}$. Dans la bibliothèque d'Amelia Rosselli, conservée aujourd'hui à Viterbe, sont présents de très nombreux livres en français, parfois annotés. Les œuvres de Proust, Rimbaud, Mallarmé, mais aussi de Lacan ou de Barthes témoignent d'un intérêt pour les classiques français ainsi que pour les sciences humaines françaises contemporaines. Le surréalisme et la poésie de Rimbaud constituent également des références constantes dans Primi scritti. La langue et la littérature françaises sont perçues comme étant inséparables, si bien qu'Amelia Rosselli se demande dans Diario in Tre Lingue : " why is French (not Engl Germ Ital.) tied to surrealism Ironique? (structure de?) / les images n'y semblent jamais sérieuses / simply because Surrealism was born in France, is now (natural ?) language ${ }^{15} »$. Toutefois, la fixation d'une langue dans son identité essentielle n'est jamais définitive. Au contraire, pour Amelia Rosselli, chaque langue se définit et existe par rapport aux autres, comme le suggère la phrase que nous avons citée en épigraphe : « fleurs are not flowers ».

11 Aussi, l'interaction du français avec les autres langues connues par Amelia Rosselli est décisive. De fait, en raison de leur appartenance commune aux langues romanes, le français et l'italien tendent plus facilement à être réunis et à interférer. Les études statistiques sur les interférences entre les langues dans les textes d'Amelia Rosselli montrent que les interférences entre français et italien sont plus fréquentes que celles entre le français et l'anglais ${ }^{16}$. Cette donnée est confirmée par les notes de traductions conservées à même les livres de la bibliothèque d'Amelia Rosselli : nous n'avons trouvé aucune note de traduction allant de l'anglais vers le français, alors que toutes les autres combinaisons sont représentées avec une fréquence variable, la préférence étant donnée à la traduction vers l'italien, qui se présente comme la langue la plus utilisée. 


\section{Un français multilingue}

12 Dans ce système de ressemblances et de différences, l'éloignement de la norme traditionnelle $d u$ français est lié en particulier à l'interférence d'autres langues. Concrètement, la voix poétique semble se trouver dans une situation instable par rapport à sa langue, comme un locuteur qui serait en apprentissage permanent. Les textes français d'Amelia Rosselli présentent notamment des figures de bégaiement, de répétition-variation, d'hésitation sur le genre des mots : «Est-elle façade (quelconque qu'elle soit) où est-il façade ? ${ }^{17}$ ». Cette phrase mime le flottement de genre sous une forme spécifiquement française: l'inversion interrogative entre le verbe et le pronom avec liaison ne peut être exprimée de la même manière en anglais ou en italien. Les particularités du français sont parodiées à dessein. L'hésitation ou la transformation du genre est une figure stylistique cruciale qui se décline chez Amelia Rosselli aussi dans les autres langues. En italien, la métathèse de genre intervient aussi au niveau des substantifs. Par exemple, dans Variazioni belliche, la forme « castelle » vient remplacer celle de « castelli ${ }^{18}$ », en ressuscitant ainsi une forme attestée en italien dialectal ou archaïque.

13 Le multilinguisme s'exprime de manière plus frappante lors d'interférences linguistiques explicites. En reprenant les catégories établies par Weinreich, ces figures consistent tout d'abord en l'insertion de mots en d'autres langues ("code-switching» ${ }^{19}$ ), comme dans cet extrait du poème en prose Le Chinois à Rome $e^{20}$ :

tu puoi capovolgere la vita

(tu peux renverser la vie)

La double phrase en italien puis en français est centrée au milieu de la page, cernée de blanc. La phrase en français, mise en valeur par les italiques, n'est pas un simple doublon, mais elle devient, grâce à la disposition typographique, un véritable sous-titre. De plus, la traduction apporte une nuance sémantique. Le verbe français « renverser» porte une violence supplémentaire par rapport à l'italien « capovolgere ».

Les changements de langue peuvent avoir lieu aussi au sein d'une même phrase et impliquer les trois langues à la fois : «La lumière s'épanouit spreads itself si spande ( $s$ 'évanouit) ${ }^{21}$ ». La phrase suit un mouvement mimétique qui suggère l'expansion même de la lumière, par une chaîne phonique en $(s) /(p)^{22}$. Elle effectue un déroulement circulaire : elle part du français, passe en anglais, puis en italien, et revient au français. Or, à travers ce passage, le sens se modifie subtilement, par la paronomase «s'épanouit/s'évanouit ». Il ne s'agit donc pas d'une traduction, mais d'une pseudo-traduction où le sens change peu à peu.

Non seulement les autres langues apparaissent dans le français, mais elles peuvent aussi se combiner lors de la création de mots inventés («code-mixing», selon la terminologie de Weinreich): par exemple "pavement», "esilaration», ou encore "fleurier ${ }^{23}$ ». Ces néologismes semblent avoir comme modèle surtout l'anglais de Joyce ou de Lewis Carroll, deux modèles explicitement cités par Amelia Rosselli, même si la formation de motsvalises n'est pas tout à fait étrangère à la langue italienne ${ }^{24}$. Comme nous le verrons ultérieurement, ces mots sont patiemment analysés par l'auteure, qui constitue un glossaire pour en expliquer l'origine.

Les différentes figures d'alternances et de mélanges linguistiques forment des chaînes de signification. Leur combinaison aboutit à un glissement de signifiants qui gouverne le mouvement du texte, en particulier dans Diario in Tre Lingue (1955-1956) ${ }^{25}$ : 
(Elle se fouilla un petit beurre gâté

gatà)

(elle se fouya (fudrà) un petit bird gâté (piccione) contrôle formel assuré. En ce sens, les fonds manuscrits de l'auteure peuvent éclairer l'histoire de ses textes et de leurs langues. Les fonds d'Amelia Rosselli - une imposante bibliothèque d'auteure ainsi qu'un fonds conséquent de brouillons manuscrits et tapuscrits - témoignent d'un long travail d'étude et de recherche ayant l'«entrelangue » comme lieu originel ${ }^{30}$.

Le travail d'écriture commence, nous y avons fait allusion précédemment, dans bibliothèque de l'auteure ${ }^{31}$. Les notes manuscrites d'Amelia Rosselli sur les livres de sa bibliothèque sont surtout des notes de traductions : mais certains livres présentent aussi des esquisses et des notes préparatoires à l'écriture. Sauf quelques exceptions significatives, les notes en français y sont peu nombreuses. Nous avons même trouvé un poème inédit et inconnu sous forme de note manuscrite dans une édition de poche des textes de Léonard de Vinci. Nous en fournissons une transcription diplomatique ${ }^{32}$ :

«C'est la nuit que l'on meurt et que l'on

résuscite

presque fous, identique aux dieux

Nus, désésperés voyage-on

au bout du monde 


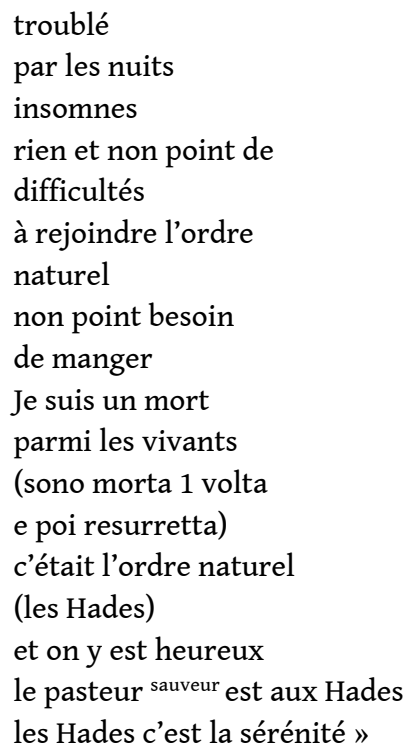

Ce texte date probablement de 1954 : telle est la date manuscrite sur la page de garde. Malgré son aspect fragmentaire et inachevé, ce brouillon est exemplaire d'une écriture qui adopte spontanément l'interférence linguistique: on remarquera l'accentuation erronée (" résuscite », « désésperés ») mais aussi l'inversion du pronom ("voyage-on ») ou encore l'adjectif hybride "insomnes » (de l'italien "insonni »). Par ailleurs, l'italien affleure également entre parenthèses, mais se trouvant réattribué au genre de l'auteure. On remarquera aussi la continuité formelle et thématique entre ce poème, publié ici pour la première fois, et les textes en français dont nous avons déjà parlé, qui datent de la même période.

Le rapprochement de ce poème et des textes ultérieurs permet alors de suivre le mûrissement de la conscience linguistique de l'auteure. En effet, les tapuscrits de plusieurs recueils d'Amelia Rosselli - qui écrivait directement à la machine - font l'objet d'interventions manuscrites concernant surtout les interférences entre les langues. C'est comme si ces points textuels avaient nécessité une attention toute particulière, donnant lieu à des repentirs, des corrections ou des annotations en marge. Par exemple, dans le texte anglais My Clothes to the Wind, 1952, l'auteure choisit de remplacer le mot cupola (un emprunt de l'italien qui est attesté en anglais) par son équivalent français, coupole, un mot qui n'est pas attesté en anglais sous forme d'emprunt, et dont l'étrangeté linguistique est donc majeure. À d'autres endroits, l'auteure glose le sens d'un mot inventé en notant son origine supposée. Ce type d'interventions manifeste le contrôle que l'auteure impose à la langue de ses textes, mais aussi la distance qui existe entre sa compétence linguistique et sa performance littéraire.

De plus, après avoir corrigé et revu ses textes, l'auteure procède également à un accompagnement éditorial. Lors de la publication de Variazioni belliche chez Garzanti, les éditeurs demandèrent à Amelia Rosselli de constituer un glossaire de ses mots inventés afin d'éclairer sa démarche poétique. Cette idée, jugée sans doute trop didactique, fut ensuite abandonnée, et le document explicatif ne fut pas inclus dans le recueil. Il fut publié bien plus $\operatorname{tard}^{33}$. Or, le même procédé est adopté pour Le Chinois à Rome, à destination cette fois d'un éditeur français. Nous avons découvert une «note pour l'éditeur ", auparavant inconnue, qui devait accompagner Le Chinois à Rome, et que nous transcrivons ici dans son intégralité ${ }^{34}$ : 


\section{Le Chinois à Rome}

note pour l'éditeur

Le récit est correct en ce qui concerne la langue en partant de la première page : puis sont inclus peu à peu des mots d'origine douteuse, ou inventée, ou de formation mi-italienne, ou, quelquefois, d'origine anglaise: ceci de façon croissante, jusqu'au chaos linguistique de la dernière page.

Page 2

Esilaration : de l'italien 'esilarare' = égayer

Page 3

métapontexes (inventé) de l'italien 'Metaponto'(ville)

couleur crémone : de l'italien 'Cremona' (ville) et 'cremisi' (couleur)

bruier: de 'bruit' (invention)

Page 4

béons : de l'italien 'beone' = ivrogne

Page 5

forchug

forchu

décolorisé : (invention)

avidvide : (invention)

Page 6

ie : anglais pour 'par exemple'

'L'Ultima Cena. Visione di Cristo e Discepoli benchè seduta alla pizzeria angolo viale Trastevere' $=$ italien pour 'Le Dernier Repas. Vision du Christ et Disciples bien que assise à la Charcuterie angle Boulevard Trastevere'.

selciato $:$ italien $=$ pavé

excrimer, coincision, circumsision, circumflexe, circonfusion

circubalation : (fusion de mots, ou inventions)

Cara Silv. (casa ecc.) - Chère Sylvaine (maison etc.)

sbouchée : de l'italien 'sboccata' = débouchée

stand $:$ anglais $=$ se tenir debout

'sembri semplicemente' : italien = tu parais simplement (seulement)

vi sono delle situazioni d'eccezione delle quali il rapporto tra guidatore e guida muta: italien =il y a des situations exceptionnelles où le rapport entre le conducteur et le guide change ('I King')

Amelia Rosselli souligne d'abord que la langue de son texte est sans erreurs, du moins au début : « correct en ce qui concerne la langue ». Toutefois, la norme linguistique est peu à peu transgressée par l'insertion de plus en plus fréquente de néologismes, décrits - non sans ironie - comme des mots « d'origine douteuse ». Mais le doute est vite dissipé : ces mots sont de «formation mi-italienne» ou quelques fois «d'origine anglaise ». Ici, une nuance est introduite entre les deux langues qui interfèrent avec le français. L'italien intervient directement dans la création de véritables mots "hybrides", tandis que l'anglais sert plutôt à des emprunts. Cette utilisation différenciée entre les deux langues correspond aussi à leur appartenance à des aires différentes : comme nous le rappelions précédemment, l'italien et le français peuvent se mélanger plus facilement car, en tant que langues romanes, elles ont davantage de racines en commun.

L'introduction de mots inventés s'intensifie au fil du texte dans un crescendo, jusqu'à arriver au " chaos linguistique de la dernière page ». Ce " chaos » est un moment rêvé ou cauchemardesque d'indifférenciation. Toutes les différences s'effondrent et cessent d'exister, entraînant en même temps la fin de toute langue. Ce moment d'abolition radical est peut-être aussi le signe d'un retour à la mythique «Ur-Sprache » qui aurait préexisté à la différenciation des langues ${ }^{35}$. Cette formule finale provoque aussi un effet de surprise et de fascination destiné à emporter l'adhésion du lecteur ou, dans ce cas, de l'éditeur. 

devancer la critique. Tout comme le glossaire qui avait été prévu pour une édition italienne, ce glossaire français ne constitue pas une étude objective ou exhaustive. En empruntant des codes scientifiques, ce dispositif est destiné avant tout à rationaliser la création poétique, à persuader le lecteur de l'originalité de la démarche, et à manifester enfin la conscience critique de l'auteure sur sa propre écriture, quitte à falsifier le procédé « philologique » (qui correspond lui-même à une construction idéale du texte, ne pouvant restituer sa réalité que de manière asymptotique).

Ainsi, cette "note pour l'éditeur» passe sous silence bien des allusions biographiques présentes dans le texte. L'auteure donne des explications de certains mots qui sont, par ailleurs, « douteuses » : ainsi, le mot « forchug » est déclaré comme provenant de l'italien, alors même que dans le texte Le Chinois à Rome il apparaît comme "forchung ", révélant une (rare) influence de l'allemand. Certaines traductions sont manifestement des adaptations, puisque le mot "pizzeria» est transformé en "charcuterie». L'auteure exhibe son laboratoire linguistique pour en prouver la validité, mais elle n'en donne pas toutes les clefs. En proposant au lecteur de parcourir à nouveau l'itinéraire de création interlinguistique, elle l'invite en fin de compte à entrer, lui aussi, dans le jeu de la création et de l'interprétation, à participer à son tour au « chaos linguistique ».

\section{Conclusion}

Après l'expérience de l'écriture plurilingue dans le Diario in tre lingue, Amelia Rosselli choisit l'italien pour son premier recueil Variazioni belliche. Le français semble devenir plus rare comme langue d'écriture, ce que confirment les fonds manuscrits: on constate une raréfaction progressive des annotations en français, que ce soit en marge des livres de sa bibliothèque ou dans ses manuscrits ${ }^{36}$. Le lien à la France, toujours présent, se détend dans la mesure où les textes français d'Amelia Rosselli n'ont jamais été publiés par un éditeur français. Ils paraissent en revanche chez Guanda, prestigieuse maison d'édition italienne, en 1980.

Amelia Rosselli n'abandonne jamais le français, qui devient une «langue dormante ${ }^{37}$ » continuant d'influencer son écriture. Ainsi, le premier poème de Variazioni belliche contient un seul mot en français, la conjonction « car », qui ressort de manière frappante dans la phrase italienne où elle est insérée : « un babelare commosso; car le foglie secche e gialle rapiscono $~_{38}$. Dans le deuxième recueil, Serie ospedaliera, apparaît un syntagme-clé : " amour je t'ai tués9 ». D'autres textes introduisent des mots de l'italien ancien qui sont également des emprunts au français, comme le mot tornasole au lieu de girasole, dans Diario ottuso (Journal obtus) ${ }^{40}$. Le troisième recueil, Documento, placé par l'auteure sous le signe de Pétrarque et de Mallarmé, connaît aussi une importante utilisation du lexique français. La persistance du français se reflète enfin dans la composition du long poème Impromptu : « Non v'è sole che non sia / lumière, (e il francese è / un par terre) ${ }^{41}$ ». Dans tous les cas, l'interférence linguistique reste le vecteur d'une influence culturelle. La légitimité acquise par les premières publications est décisive: elle permet à Amelia Rosselli d'imposer le multilinguisme de son écriture de manière de plus en plus forte.

31 Aujourd'hui, la réception critique d'Amelia Rosselli, marquée par de nombreux malentendus, évolue positivement en Italie et à l'étranger, comme en témoignent les traductions anglaises (War variations ${ }^{42}$ ) et françaises (Impromptu ${ }^{43}$ et Sleep $^{44}$ par Jean- 
Charles Vegliante; Variations de guerre, par Marie Fabre ${ }^{45}$ ). Ces traductions sont peut-être le meilleur signe d'une nouvelle approche des œuvres d'Amelia Rosselli : en traduisant un texte plurilingue, elles accomplissent et donnent à lire le mouvement entre les langues qui a toujours été le sien.

\section{BIBLIOGRAPHIE}

Agosti, Stefano, « La competenza associativa di Amelia Rosselli », (La Compétence associative d'Amelia Rosselli) dans Poesia Italiana Contemporanea, Milano, Bompiani, 1978, p. 133-151.

Anokhina, Olga, « Vladimir Nabokov : du style et des langues », in St. Bikialo et S. Pétillon (éds.), La Licorne, numéro spécial : « Dans l'atelier du style. Du manuscrit à l'œuvre publiée », 2012, n 98, Rennes, Presses Universitaires de Rennes, p. 211-220.

Baldacci, Alessandro, Amelia Rosselli, Roma-Bari, Laterza, 2007.

Bisanti, Tatiana, L'opera plurilingue di Amelia Rosselli, (L'Euvre plurilingue d'Amelia Rosselli), Pisa, ETS, 2007.

De March, Silvia, Amelia Rosselli tra poesia e storia, (Amelia Rosselli entre poésie et histoire) introduzione di Andrea Zanzotto, Napoli, L’Ancora del mediterraneo, 2006.

Derrida, Jacques, Le Monolinguisme de l'autre ou la prothèse de l'origine, Paris, Galilée, 1996.

D’Iorio, Paolo et Daniel, Ferrer (éds.), Bibliothèques d'écrivains, Paris, CNRS, 2001.

Griggs, Peter, Perspective sociocognitive sur l'apprentissage des langues étrangères, Paris, l'Harmattan, 2007.

La Penna, Daniela, « La mente interlinguistica. Strategie dell'interferenza nell'opera trilingue di Amelia Rosselli » (L'esprit interlinguistique. Stratégie de l'interférence dans l'œuvre trilingue d'Amelia Rosselli), dans Vincenzo Orioles et Furio Brugnolo (éds.), Eteroglossia e plurilinguismo letterario, vol. II, Plurilinguismo e letteratura, Atti del XXVIII convegno interuniversitario di Bressanone (6-9 luglio 2000), Roma, Il Calamo, 2002, p. 397-415.

Manera, Manuela, «L'“ydioma tripharium” di Amelia Rosselli. Ricognizioni linguistiche » (L'“Y dioma tripharium" d'Amelia Rosselli. Observations linguistiques), dans Lingua e Stile, Rivista di storia della lingua italiana, anno XXXVIII, n.2, dicembre 2003, p. 233-267.

Migliorini, Bruno, Storia della lingua italiana (Histoire de la langue italienne), Milano, Bompiani, 2007. Rosselli, Amelia Le poesie, a cura di Emmanuela Tandello, prefazione di Giovanni Giudici, Milano, Garzanti, 1997.

Cette édition contient les textes suivants :

Primi scritti (Premiers écrits), 1952-1963.

Variazioni belliche (Variations de guerre), 1959-1963.

Spazi metrici (Espaces métriques),1963-1965.

La libellula (La Libellule), 1958.

Serie ospedaliera (Série hospitalière), 1963-1965. 
Documento (Document), 1966--1973.

Impromptu (Impromptu), 1981.

Rosselli, Amelia, Diario ottuso (Journal obtus) (1954-68), Roma, IBN, 1990.

Rosselli, Amelia, Sleep. Poesie in inglese (Sleep. Poésies en anglais), traduction en italien de Emmanuela Tandello, Milano, Garzanti, 1992.

Rosselli, Amelia, Una scrittura plurale. Saggi e interventi critici, a cura di Francesca Caputo, Novara, Interlinea, 2004.

Rosselli, Amelia, È vostra la vita che ho perso, conversazioni e interviste 1964-1995 (C'est votre vie que j'ai perdue, conversations et entretiens), a cura di Monica Venturini et Silvia De March, Firenze, Le Lettere, 2010.

Rosselli, Amelia, Impromptu, traduction en français de Jean-Charles Vegliante, Paris, La Tour de Babel, 1987, maintenant dans Impromptu, a trilingual édition (by G.M. Annovì), Montreal, Guernica, sous presse.

Rosselli, Amelia, «Sleep - choix de poèmes », Jean-Charles Vegliante, in $\operatorname{Doc}(k)_{s}, \mathrm{n}^{\circ} 1$, été-automne, 1991.

Rosselli, Amelia, War variations, traduction en anglais Lucia Re et Paul Vangelisti, København \& Los Angeles, Green Integer, 2005.

Rosselli, Amelia, Variations de guerre, traduction en français de Marie Fabre, Paris, Ypsilon, 2012.

Sciarrino, Emilio, « Les Langues d'Amelia Rosselli ", dans Revue critique de fixxion française contemporaine, $\mathrm{n}^{\circ} 3$ : L'Écrivain devant les langues, Dominique Combe et Michel Murat (éds.), 2011. Steiner, Georges, Après Babel, traduit de l'anglais par Lucienne Lotringer, Albin Michel, Paris, 1978.

Tzara, Tristan, Dada est tatou. Tout est Dada., Paris, Flammarion, 1996.

Vegliante, Jean-Charles, Ungaretti entre les langues, Paris, Centre de recherches sur l'Italie moderne et contemporaine, université de la Sorbonne Nouvelle-Paris 3, 1987.

Vegliante, Jean-Charles, Poésie entre les langues. Amelia Rosselli, Paris, prépublication CIRCE, 1994 : trad. fr. et présentation par Jean-Charles Vegliante.

Vegliante, Jean-Charles, D'écrire la traduction, Paris, Presses de la Sorbonne, 1996.

Vegliante, Jean-Charles, « Tradurre /l'entrelangue/ » dans Per Edoardo Sanguineti, lavori in corso, Firenze, F. Cesati, 2012.

Weinreich, Uriel, Languages in Contact: findings and problems, (Langues en contact : découvertes et problèmes), New York, Linguistic Circle of New York, 1953.

\section{NOTES}

1. Amelia Rosselli, «Diario in Tre Lingue » (Journal en Trois Langues), dans Primi scritti (Premiers écrits), Milano, Guanda, 1980, repris dans Le poesie (Les poésies), Milano, Garzanti, 1997, p. 91.

2. Jean-Charles Vegliante, Poésie entre les langues. Amelia Rosselli, Paris, prépublication CIRCE, 1994 : tr. fr. et présentation par Jean-Charles Vegliante.

3. Manuela Manera, "L"'ydioma tripharium” di Amelia Rosselli. Ricognizioni linguistiche » (L'« ydioma tripharium » d'Amelia Rosselli. Observations linguistiques), dans Lingua e Stile, Rivista di storia della lingua italiana, anno XXXVIII, n. 2, dicembre 2003, p. 233-267 ; voir aussi Daniela La 
Penna, «La mente interlinguistica. Strategie dell'interferenza nell'opera trilingue di Amelia Rosselli » (L'esprit interlinguistique. Stratégie de l'interférence dans l'œuvre trilingue d'Amelia Rosselli), dans Vincenzo Orioles et Furio Brugnolo (éds.), Eteroglossia e plurilinguismo letterario, vol. II, Plurilinguismo e letteratura, Atti del XXVIII convegno interuniversitario di Bressanone (6-9 luglio 2000), Roma, Il Calamo, 2002, p 397-415.

4. Ces textes sont rassemblés dans le recueil suivant: Amelia Rosselli, Primi scritti, op. cit.

5. Amelia Rosselli, Variazioni belliche (Variations de guerre), Milano, Garzanti, 1964, puis dans Le poesie, op. cit.

6. Amelia Rosselli, Sleep. Poesie in inglese (Sleep. Poésies en anglais), traduction en italien de Emmanuela Tandello, Milano, Garzanti, 1992.

7. Voir en particulier la synthèse d'Alessandro Baldacci, comprenant un chapitre final sur l'histoire de la critique d'Amelia Rosselli : Alessandro Baldacci, Amelia Rosselli, Roma-Bari, Laterza, 2007.

8. À ce propos, voir l'entretien avec la romancière et militante féministe Dacia Maraini, dans Amelia Rosselli, È vostra la vita che ho perso, conversazioni e interviste 1964-1995 (C'est votre vie que j'ai perdu, conversations et entretiens), a cura di Monica Venturini et Silvia De March, Firenze, Le Lettere, 2010, p. 9.

9. Jacques Derrida, Le monolinguisme de l'autre ou la prothèse de l'origine, Paris, Galilée, 1996.

10. Amelia Rosselli, «Figli della guerra » (Fils de la guerre), entretien avec Paola Zacometti, dans È vostra la vita che ho perso, conversazioni e interviste 1964-1995, op. cit., p. 116.

11. "A Parigi abbiamo sempre parlato un miscuglio di lingue: italiano con il babbo e la mamma, inglese con le bambinaie inglesi, francese a scuola " (ici et plus loin notre traduction). Cité par Silvia De March, Amelia Rosselli tra poesia e storia, (Amelia Rosselli entre poésie et histoire) introduzione di Andrea Zanzotto, Napoli, L'Ancora del mediterraneo, 2006, p. 23.

12. "Si può dire che in qualche modo non ha mai avuto una "lingua madre" ", Ibid.

13. Amelia Rosselli, Sanatorio 1954, dans Le poesie, op. cit., p. 23.

14. L'influence $d u$ français est perceptible chez d'autres auteurs italiens de la génération précédente (voir Jean-Charles Vegliante, Ungaretti entre les langues, Paris, Centre de recherches sur l'Italie moderne et contemporaine, université de la Sorbonne Nouvelle-Paris 3,1987) et de la même période (Sanguineti, Arbasino).

15. Amelia Rosselli, « Diario in Tre Lingue », dans Le poesie, op. cit., p 98.

16. Voir les analyses statistiques de Tatiana Bisanti, L'opera plurilingue di Amelia Rosselli, (L'œuvre plurilingue d'Amelia Rosselli), Pisa, ETS, 2007. Par exemple, pour Diario in Tre Lingue, Tatiana Bisanti relève 71 passages de l'italien à l'anglais et 69 passages de l'anglais à l'italien, contre 40 passages de l'italien au français et 41 passages du français à l'italien, et seulement 33 passages du français à l'anglais, et autant de passages dans le sens inverse, ce qui représente en tout 110 passages vers l'italien, 107 passages vers l'anglais et 74 vers le français.

17. Amelia Rosselli, « Le Chinois à Rome ", dans Le poesie, op. cit., p. 51.

18. Amelia Rosselli, «Variazioni belliche », dans Le poesie, op. cit., p. 225.

19. Uriel Weinreich, Languages in Contact : findings and problems, (Langues en contact : découvertes et problèmes), New York, Linguistic Circle of New York, 1953.

20. Amelia Rosselli, « Le Chinois à Rome », dans Le poesie, op. cit., p. 53.

21. Ibid., nous soulignons.

22. Cet attachement aux effets sonores se retrouve également chez Nabokov lors de l'écriture ou la traduction de ses œuvres en anglais. A ce sujet, voir Olga Anokhina, « Vladimir Nabokov : du style et des langues », in St. Bikialo et S. Pétillon (éds.), La Licorne, numéro spécial « Dans l'atelier du style. Du manuscrit à l'œuvre publiée », 2012, n 98, Rennes, Presses Universitaires de Rennes, p. 211-220.

23. Ibid., p. 49-51. 
24. Selon Bruno Migliorini, le mot-valise a eu une certaine fortune dans l'italien du $\mathrm{XVI}^{\mathrm{e}}$ siècle, même s'il est considéré comme un "caprice stylistique» plutôt que comme un véritable «enrichissement du lexique». Bruno Migliorini, Storia della lingua italiana (Histoire de la langue italienne), Milano, Bompiani, 2007, p. 439-440.

25. Amelia Rosselli, « Diario in Tre Lingue », dans Le poesie, op. cit., p. 79.

26. Selon l'étude inspirée de la psychanalyse proposée par Stefano Agosti, «La competenza associativa di Amelia Rosselli », (La compétence associative d'Amelia Rosselli) dans Poesia Italiana Contemporanea, Milano, Bompiani, 1978, p. 133-151.

27. Tristan Tzara, Dada est tatou. Tout est Dada., Paris, Flammarion, 1996.

28. À propos du rapport secret entre les langues et du silence consubstantiel à leur origine, voir Jean-Charles Vegliante, "Du silence dans les langues ", dans Sigila, revue transdisciplinaire francoportugaise sur le secret, $\mathrm{n}^{\circ} 23$, printemps-été 2009, p. 173-184.

29. Amelia Rosselli, Le Chinois à Rome, dans Le poesie, op. cit., p. 51 (nous soulignons).

30. Sur la notion d'« entre-langue " et sur l'intérêt des traces manuscrites qui en témoignent, voir Jean-Charles Vegliante, D'écrire la traduction, Paris, Presses de la Sorbonne, 1996, p. 57 et voir à présent son «Tradurre /l'entrelangue » dans Per Edoardo Sanguineti, lavori in corso, Firenze, F. Cesati, 2012.

31. Comme toute bibliothèque d'auteur, celle-ci constitue à la fois un lieu de mémoire des lectures et un laboratoire d'écriture: voir Paolo D'Iorio et Daniel Ferrer (éds.), Bibliothèques d'écrivains, Paris, CNRS, 2001.

32. Leonardo Da Vinci, Tutti gli scritti, vol. I, Scritti letterari, éd. Augusto Marinoni, Milano, Rizzoli, 1952, p. 260, 262-263. Le livre, conservé dans le fonds Rosselli de la bibliothèque de l'Université de Viterbe, porte la cote FAR 2354.

33. Amelia Rosselli, «Glossarietto esplicativo per Variazioni belliche» (Petit glossaire explicatif pour Variations de guerre), dans Una scrittura plurale, saggi e interventi critici, a cura di Francesca Caputo, Novara, Interlinea, 2004, p. 69.

34. Cette lettre a été publiée pour la première fois dans notre article «Les langues d'Amelia Rosselli ", dans Revue critique de fixxion française contemporaine, $\mathrm{n}^{\circ} 3$, L'écrivain devant les langues, Dominique Combe et Michel Murat (éds.), 2011.

35. Georges Steiner, Après Babel, traduit de l'anglais par Lucienne Lotringer, Paris, Albin Michel, 1978 , p. 69.

36. Dans les années 1950 on pouvait trouver des textes italiens traduits vers le français (Montale): or cette situation s'inverse au cours des années 1960, où les textes français sont traduits uniquement vers l'italien (Mallarmé et Valéry).

37. Le concept de « langue dormante » n'a pas encore une étendue très définie. Pour certains, la « langue dormante est emmagasinée en mémoire à long terme mais ne joue aucun rôle dans le traitement en cours, ne pouvant être activée qu'à un coût cognitif élevé " (Peter Griggs, Perspective sociocognitive sur l'apprentissage des langues étrangères, Paris, l'Harmattan, 2007, p. 96).

38. Amelia Rosselli, « Variazioni belliche », dans Le poesie, op. cit., p. 163 et 165 (nous soulignons).

39. Amelia Rosselli, « Serie ospedaliera », dans Le poesie, op. cit., p. 360.

40. Amelia Rosselli, Diario ottuso (1969), Roma, IBN, 1990, p. 43. Le mot tornasole (emprunt au français tournesol, datant $\mathrm{du} \mathrm{XIV}^{\mathrm{e}}$ siècle) est bien plus rare que girasole.

41. Amelia Rosselli, Impromptu, dans Le poesie, op. cit.

42. Amelia Rosselli, War variations, trad. en anglais Lucia Re et Paul Vangelisti, København \& Los Angeles, Green Integer, 2005.

43. Amelia Rosselli, Impromptu, trad. en français de Jean-Charles Vegliante, Paris, La Tour de Babel, 1987, puis Impromptu, a trilingual édition (by G.M. Annovì), Montreal, Guernica, sous presse.

44. Amelia Rosselli, Sleep - choix de poèmes, trad. en français Jean-Charles Vegliante, dans Doc $(k)$ s, $\mathrm{n}^{\circ}$ 1, été-automne, 1991.

45. Amelia Rosselli, Variations de guerre, trad. en français de Marie Fabre, Paris, Ypsilon, 2012. 


\section{RÉSUMÉS}

Cet article étudie les textes français d'Amelia Rosselli (1930-1996), auteure trilingue et voix majeure de la poésie italienne du $\mathrm{xx}^{\mathrm{e}}$ siècle. Il analyse la situation de la langue française dans son œuvre, puis précise le lien du français aux autres langues - l'anglais et l'italien -, qui interagissent dans une forte créativité linguistique. En ce sens, l'étude de manuscrits inédits révèle la genèse d'une poésie multilingue située entre compétence intuitive et performance structurée.

This paper examines the French texts of Amelia Rosselli (1930-1996), a trilingual author and a major voice of twentieth-century Italian poetry. It analyzes the position of French language in her works, then it specifies the relationship of French to the other languages, English and Italian, all interacting in a strong linguistic creativity. Thus, the study of unpublished manuscripts reveals the genesis of a multilingual poetry that lies between intuitive competence and structured performance.

\section{AUTEUR}

\section{EMILIO SCIARRINO}

Université Paris 3-Sorbonne Nouvelle 